\title{
Reduced-Complexity Near-Maximum-Likelihood Detection for Decision Feedback Assisted Space-Time Equalization
}

\author{
A. Wolfgang, J. Akhtman, S. Chen, and L. Hanzo
}

\begin{abstract}
A novel Decision-Feedback (DF) aided reduced complexity Maximum Likelihood (ML) Space-Time Equalizer (STE) designed for a single-carrier system is introduced. Two different methods of incorporating DF into the recursive tree search based receiver structure are proposed for allowing detection at a moderate computational cost. Additionally, a further complexity reduction scheme is proposed, which exploits the specific characteristics of both the wide-band channel and the proposed DF-STE. In comparison to the DF-STE not benefiting from this complexity reduction, the proposed detector is capable of reducing the complexity by several orders of magnitude. More quantatively, for the specific rank-deficient system considered, which detected the signal transmitted from four transmit antennas with the aid of two receive antennas, the complexity might be reduced by a factor of 100 at low Signal-to-Noise Ratios (SNRs) without noticeable performance degradation. By contrast, at higher SNRs a complexity reduction of a factor of 10 might be achieved, depending on the tolerable performance degradation.
\end{abstract}

Index Terms-Space-time equalization, single-carrier, sphere decoding, decision feedback.

\section{INTRODUCTION}

$\mathbf{I}$ $\mathrm{N}$ the context of Multiple-Input Multiple-Output (MIMO) systems [1] four main transmitter and receiver designs employing different detection strategies have been proposed. More specifically, space-time block- and trellis coding are designed for achieving both transmit and receive diversity gains [2]. By contrast, Spatial Division Multiple Access (SDMA) [3] aims for increasing the number of users supported by differentiating them with the aid of their unique, userspecific Channel Impulse Responses (CIR), despite communicating in the same bandwidth. Similarly, Spatial Division Multiplexing (SDM) [4] differentiates a number of antennaspecific signals of a given user with the aid of their antennaspecific CIRs for the sake of increasing a single user's throughput. Finally, beamforming supports multiple users with the aid of angularly selective beams [5] and in the context of wideband dispersive channels the beamforming receiver is often referred to as a Space-Time Equalizer (STE). All the different designs aim for maintaining an infinitesimally low error rate over the wireless channel at a data rate approaching the channel's capacity [6], [7].

This contribution primarily focuses on different realizations of detection strategies invoked for single-carrier MIMO systems operating in a dispersive propagation environment. This

Manuscript received December 1, 2005; revised September 1, 2006; accepted December 1, 2006. The associate editor coordinating the review of this paper and approving it for publication was $\mathrm{H}$. Xu. The financial support of the EU under the auspices of the Phoenix and Newcom projects is gratefully acknowledged. The authors are also grateful to their colleagues for the enlightenment gained within the Phoenix consortium.

The authors are with the School of ECS, Univ. of Southampton, SO17 1BJ, UK (e-mail: \{aw03r, yja02r, sqc, lh\}@ecs.soton.ac.uk).

Digital Object Identifier 10.1109/TWC.2007.05959. receiver is also known as a STE. Well established STE techniques are the Minimum Mean Squared Error (MMSE) [8] and the Maximum Likelihood (ML) or the Bayesian algorithms. While the MMSE detector has an attractively low complexity, it is generally outperformed by both the ML and the Bayesian detector.

Recently there has been a whole set of proposals on low complexity detectors designed for narrowband MIMO systems based on the so-called Sphere Decoder (SD) first proposed in [9] and applied to diverse detection problems in [10], [11], which all approach the performance of the ML detector. More recently a novel tree-search based reduced complexity ML detector designed for Orthogonal Frequency Division Multiplexing (OFDM) [3] systems has been introduced [4], which is referred to as the Optimized Hierarchical Recursive Search Algorithm (OHRSA).

The novel contribution of this paper is that we extend the applicability of the OHRSA to synchronous single-carrier MIMO systems operating in a dispersive environment. Directly applying the OHRSA, which was originally designed for non-dispersive OFDM sub-channels to a wideband singlecarrier system does have the potential of achieving the ML detector's performance. However, employing the OHRSA in an Inter-Symbol-Interference (ISI) contaminated wideband single-carrier environment involves the detection of both the desired and undesired ISI-induced symbols engendered by the dispersive channel, which imposes an increased computational complexity. In order to reduce the computational complexity, we firstly introduce two different methods for incorporating Decision Feedback (DF) in the proposed receiver and secondly, introduce a novel method for truncating certain levels of the search-tree. In contrast to [12], where a complexity reduction scheme was proposed for tree-search based algorithms, which operated on the basis of not evaluating certain subtrees, the method proposed in this paper aims for discarding complete hierarchical levels of the search-tree.

The remainder of this paper is organized as follows. In Section II the basic system model describing the proposed STE is introduced. The system model is used in Section III for introducing the basic concept of the OHRSA, while in Section IV the different ways of incorporating DF in the OHRSA are discussed. The proposed algorithm is then further modified in Section V, in order to accommodate the specific properties of a single-carrier wideband system, which results in a considerable complexity reduction at a negligible performance degradation. Simulation results are given in Section VI. Finally, in Section VII we offer our conclusions.

\section{SySTEM MOdEL}

The system considered is characterized by a single transmitter, employing an $M$-element transmit antenna array and 


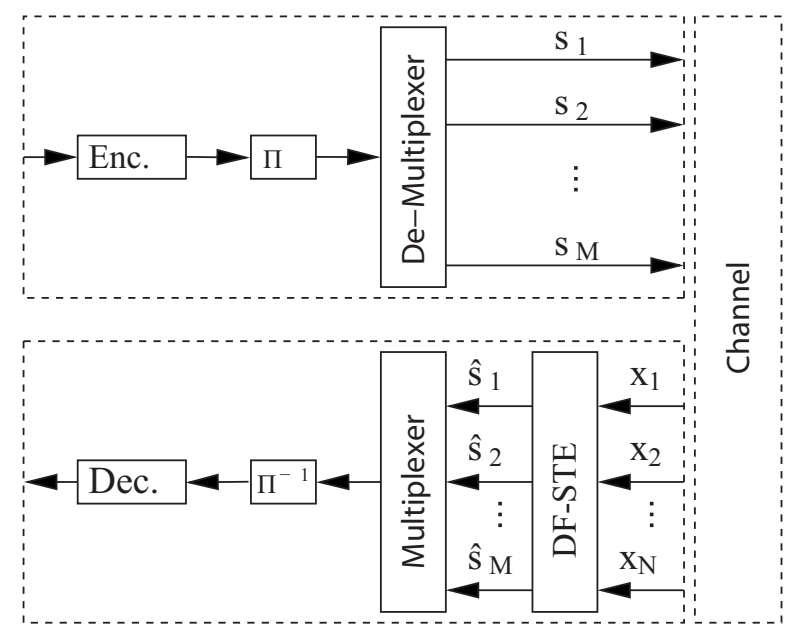

Fig. 1. System model.

a receiver, which has $N$ number of Antenna Elements (AEs). At the transmitter the input bitstream is channel encoded at a code-rate $R$ and transmitted using $M$ different transmit AEs, as illustrated in Figure 1. The modulated symbols are transmitted over a frequency selective fading channel having a symbol-spaced Channel Impulse Response (CIR) characterized by the channel coefficients $h_{n m, l}$. The CIR coefficient $h_{n m, l}$ represents the complex-valued channel gain of the $l^{\text {th }}$ multi-path component of the channel between the $m^{\text {th }}$ transmit $\mathrm{AE}$ and the $n^{\text {th }}$ receiver AE. Given the transmitted symbol $s_{m}(k)$, which is associated with transmit $\mathrm{AE} m$, the output signal of the $n^{\text {th }} \mathrm{AE}$ of the $\mathrm{BS}$ receiver at time instant $k$ can be written as

$$
x_{n}(k)=\sum_{m=1}^{M} \sum_{l=1}^{L} h_{n m, l} s_{m}(k-l+1)+\eta_{n}(k) .
$$

Furthermore, $L$ is the number of symbol-spaced multi-path components and $\eta_{n}(k)$ is the complex-valued Additive White Gaussian Noise (AWGN) having a variance of $E\left[\left|\eta_{n}(k)\right|^{2}\right]=$ $2 \sigma_{\mathrm{n}}^{2}$.

Under the assumption of perfectly synchronized transmit AEs the relation between the transmitted signal and the channel's output for channel tap $l$ is described by an $(N \times M)$ dimensional matrix $\boldsymbol{H}_{l}$, where the $(n, m)^{\text {th }}$ element of the matrix is given by $h_{n m, l}$.

Considering a finite-length STE having a feed-forward order of $N_{\mathrm{ff}}$ and operating at a decision delay $\Delta$ as defined in [13], the super-matrix $\mathbf{H}$, which represents the total system is obtained by concatenating the $(N \times M)$-dimensional matrices $\boldsymbol{H}_{l}$, yielding:

$$
\mathbf{H}=\left[\begin{array}{cccccc}
\boldsymbol{H}_{1} & \cdots & \boldsymbol{H}_{L} & 0 & \cdots & 0 \\
0 & \boldsymbol{H}_{1} & \cdots & \boldsymbol{H}_{L} & \ddots & \vdots \\
\vdots & \ddots & \ddots & \ddots & \ddots & 0 \\
0 & \cdots & 0 & \boldsymbol{H}_{1} & \cdots & \boldsymbol{H}_{L}
\end{array}\right]
$$

Let us denote the $N$-element channel output vector as $\boldsymbol{x}(k)$. Then the channel's output formulated as the super-vector

$$
\begin{aligned}
& \mathbf{x}(k)=\left[\boldsymbol{x}(k)^{T} \ldots \boldsymbol{x}\left(k-N_{\mathrm{ff}}+1\right)^{T}\right]^{T} \text { can be expressed as } \\
& \mathbf{x}(k)=\mathbf{H}\left[\boldsymbol{s}(k)^{T}, \ldots, \boldsymbol{s}\left(k-L-N_{\mathrm{ff}}+2\right)^{T}\right]^{T} \\
&+\left[\boldsymbol{\eta}(k)^{T}, \ldots, \boldsymbol{\eta}\left(k-N_{\mathrm{ff}}+1\right)^{T}\right]^{T} \\
&= \mathbf{H s}(k)+\boldsymbol{\eta}(k) \\
&= \check{\mathbf{x}}(k)+\boldsymbol{\eta}(k),
\end{aligned}
$$

where $\boldsymbol{s}(k)=\left[s_{1}(k), \ldots, s_{M}(k)\right]^{T}$ is a column vector containing the symbols transmitted by the $M$ AEs and $\boldsymbol{\eta}(k)=$ $\left[\eta_{1}(k), \ldots, \eta_{N}(k)\right]^{T}$. For notational simplicity the time-index $k$ will be dropped, where this is possible without ambiguity. When referring to a delayed vector, such as for example $\boldsymbol{s}(k-\Delta)$, this is indicated by $\boldsymbol{s}_{\Delta+1}$, which suggests that $\boldsymbol{s}_{\Delta+1}$ is the $(\Delta+1)^{\text {th }}$ subvector of the super-vector $\mathbf{s}(k)$ defined in Equation (2).

\section{The ML Detector}

In this section the OHRSA designed for the detection of the transmitted signal [4] is highlighted. The ML detection of the transmitted signal vector is given by

$$
\hat{\mathbf{s}}=\arg \min _{\check{\mathbf{s}} \in \mathcal{S}}\left\{\|\mathbf{x}-\mathbf{H} \check{\mathbf{s}}\|^{2}\right\},
$$

where $\mathcal{S}$ is the set of potentially transmitted symbol vectors $\mathbf{s}$. Note that in this formulation the super-vector $\mathbf{s}$ is detected, rather than only the subvector of interest, namely $s_{\Delta+1}$. It was shown in [4] that the solution to the problem defined by Equation (3) is identical to solving

$$
\hat{\mathbf{s}}=\arg \min _{\check{\mathbf{s}} \in \mathcal{S}}\left\|\mathbf{U}\left(\check{\mathbf{s}}-\hat{\mathbf{s}}_{\mathrm{MMSE}}\right)\right\|^{2},
$$

where $\mathbf{U}$ is an upper triangular matrix obtained with the aid of Cholskey factorization of the received signals' covariance matrix and $\hat{\mathbf{s}}_{\mathrm{MMSE}}$ is the MMSE solution, as discussed in [4]. It was also shown in [4] that solving Equation (4) is identical to solving

$$
J_{i}\left(\check{\mathbf{s}}_{i}\right)=J_{i+1}\left(\check{\mathbf{s}}_{i+1}\right)+\phi_{i}\left(\check{\mathbf{s}}_{i}\right), \quad i=N_{\mathrm{s}}-1, \ldots, 1,
$$

where $\check{\mathbf{s}}_{i}=\left[\check{s}_{i}, \ldots, \check{s}_{N_{\mathrm{s}}}\right]^{T}, N_{\mathrm{s}}=M\left(N_{\mathrm{ff}}+L-1\right)$ is the number of symbols in s considered by the OHRSA and $\phi_{i}\left(\check{\mathbf{s}}_{i}\right)$ may be expressed as [4]

$$
\phi_{i}\left(\check{\mathbf{s}}_{i}\right)=\left|u_{i i}\left(\check{s}_{i}-\hat{s}_{\mathrm{MMSE}, i}\right)+\sum_{j=i+1}^{N_{\mathrm{s}}} u_{i j}\left(\check{s}_{j}-\hat{s}_{\mathrm{MMSE}, j}\right)\right|^{2},
$$

where $u_{i j}$ is the $(i, j)^{\text {th }}$ element of $\mathbf{U}$. Equation (4) can be efficiently solved using a binary search-tree, as outlined in [4].

In order to ensure that the algorithm operates efficiently, it is advisable to first reorder the columns of the CIR matrix in ascending order according to the energy of the columns. This will result in a "best-first" detection strategy, as outlined in [3] since the highest-energy columns correspond to the most reliably received signal componenets. For Binary Phase Shift Keying (BPSK) modulated signals, the columns of the CIR matrix $\mathbf{H}$ are therefore re-arranged, so that the re-ordered CIR matrix $\mathbf{H}^{(o)}$ satisfies

$$
\left\|\left(\mathbf{H}^{(o)}\right)_{1}\right\|^{2} \leq\left\|\left(\mathbf{H}^{(o)}\right)_{2}\right\|^{2} \leq \ldots \leq\left\|\left(\mathbf{H}^{(o)}\right)_{N_{\mathrm{s}}}\right\|^{2},
$$


where $\left\|\left(\mathbf{H}^{(o)}\right)_{i}\right\|^{2}$ indicates the energy or squared norm of the $i^{\text {th }}$ column of $\mathbf{H}^{(o)}$. In order to apply this scheme for detecting $\mathcal{M}$-level Quadrature Amplitude Modulated ( $\mathcal{M}$-QAM) signals, when encountering complex-valued CIR matrices, the system is decomposed into a real-valued binary system, as discussed for example in [10], [4].

\section{INCORPORATING DECISION FEEDBACK}

The performance of both linear and non-linear STEs can be enhanced by incorporating a decision feedback structure [13] in their receiver. In addition to the feed-forward order $N_{\mathrm{ff}}$ and the decision delay parameter $\Delta$ of the STE, we introduce the decision feedback order $N_{\mathrm{fb}}$. Note that the oldest symbol vector, which still influences the detected symbol $\hat{\boldsymbol{s}}_{\Delta+1}$ is $\boldsymbol{s}_{N_{\mathrm{ff}}+L-1}$. Furthermore, the oldest feedback symbol vector is $\boldsymbol{s}_{\Delta+N_{\mathrm{fb}}+1}$. Without loss of generality, we therefore chose a DF order of $N_{\mathrm{fb}}=N_{\mathrm{ff}}+L-2-\Delta$ for the characterization of the proposed DF aided STE.

In order to develop the feedback assisted OHRSA, the CIR matrix $\mathbf{H}$ is partitioned into two sub-matrices [13] as follows:

$$
\mathbf{H}=\left[\begin{array}{ll}
\mathbf{H}_{1} & \mathbf{H}_{2}
\end{array}\right],
$$

where $\mathbf{H}_{1}$ hosts the first $M(\Delta+1)$ number of columns of $\mathbf{H}$, while $\mathbf{H}_{2}$ represents the last $M N_{\mathrm{fb}}$ columns in $\mathbf{H}$. The attainable complexity reduction and the achievable performance gain associated with DF accrues from the fact that the previously received symbols of all transmitters have already been decided upon and hence their contribution imposed on the phasor constellation at the output of the dispersive channel may be eliminated. The number of binary symbols considered by the OHRSA invoked in the DF-STE is given as $N_{\mathrm{s}}=$ $\log _{2}(\mathcal{M}) M(\Delta+1)$, where $\mathcal{M}$ is the number of modulation levels.

\section{A. Space-Translation Based Complexity Reduction}

Similar to other STE implementions [14], DF might be incorporated by performing a so-called space-translation [13], [14] on the channel output. Equation (2) may be re-written as

$$
\mathbf{x}=\mathbf{H}_{1} \mathbf{s}_{1}+\mathbf{H}_{2} \mathbf{s}_{2}+\boldsymbol{\eta},
$$

where $\mathbf{s}_{1}=\left[\boldsymbol{s}_{1}^{T} \ldots \boldsymbol{s}_{\Delta+1}^{T}\right]^{T}$ indicates the symbols in the feed-forward shift register and $\mathbf{s}_{2}=$ $\left[\boldsymbol{s}_{\Delta+2}^{T} \ldots \boldsymbol{s}_{\Delta+N_{\mathrm{fb}}+1}^{T}\right]^{T}$ denotes the symbols in the feedback register.

Equation (9) may be interpreted as a space translation [13], [14], where a received signal vector $\mathbf{x}$ is translated into the new observation space $\mathbf{r}$ by eliminating the phasor-points corresponding to the product of the DF sequence $s_{2}$ and the CIR matrix $\mathbf{H}_{2}$. The related space translation is described by $\mathbf{r}=\mathbf{H}_{1} \mathbf{s}_{1}+\boldsymbol{\eta}=\mathbf{x}-\mathbf{H}_{2} \mathbf{s}_{2}$.

The DF assisted receiver first translates the received signal vector $\mathbf{x}$ into the translated space $\mathbf{r}$ and the detector then operates on the translated received signal vector $\mathbf{r}$, assuming that $\mathbf{H}_{1}$ was the observed CIR matrix. Finally, the OHRSA may be applied, as outlined in Section III.

\section{B. Tree-Based Complexity Reduction}

A second way of incorporating DF in the proposed receiver structure is to include the specific symbols, which have already been decided upon in the top section of the search-tree. This is achieved by modifying the 'best-first' CIR-matrix column re-ordering of Equation (7) by ensuring that the CIR submatrix $\mathbf{H}_{2}$ associated with the DF symbols retains its position within $\mathbf{H}$ during the re-ordering process, yielding $\mathbf{H}^{(o)}=$ $\left[\mathbf{H}_{1}^{(o)} \mathbf{H}_{2}^{(o)}\right]$. Accordingly, DF symbols will now appear at the top of the search-tree, which was arranged from top to bottom according to the decending order of the energy or squared norm of the associated CIR-matrix columns.

When identifying the ML solution under the assumption of correct DF symbols with the aid of the OHRSA [4], $N_{\mathrm{fb}} M$ number of symbols within the hypothesized solution $\check{\text { s are set }}$ to the already 'sliced' symbols hosted by the DF sequence $\mathbf{s}_{2}$. This measure reduces the size of the search space and the associated computational complexity imposed by searching through the detection space.

\section{TRUNCATED SEARCH}

In contrast to the employment of the OHRSA for the detection of narrowband OFDM subcarriers [4], the detection of all symbols in the vector $\mathbf{s}$ is not required in the context of wideband systems, since we mentioned earlier that we are only interested in the detection of the desired subvector $\boldsymbol{s}_{\Delta+1}$ and we specifically want to eliminate the dispersion-induced low-energy symbols.

An attractive way of exploiting this potential complexity reduction is to arrange the columns of the CIR matrix $\mathbf{H}$ not only according to the ascending order of their squared norm or energy, as suggested in [4], but also ensuring that the columns of specific interest corresponding to the originally transmitted symbols $s_{\Delta+1}$ appear at the end of the ordered CIR matrix $\mathbf{H}^{(o)}$. In other words, we aim for ensuring that the bits associated with the desired subvector $\boldsymbol{s}_{\Delta+1}$ appear at the top of the search tree and are therefore detected first. ${ }^{1}$

Let us now assume that in order to incorporate a DF structure in the receiver, the space-translation approach of Section IV is considered. The resultant new reordering scheme invoked for beneficially arranging the CIR matrix $\mathbf{H}_{1}$ can then be divided into two steps as follows:

1) Re-order the last $M$ number of columns of the feedforward CIR matrix given by

$$
\left(\mathbf{H}_{1}\right)_{i} i \in\{\Delta M+1, \ldots,(\Delta+1) M\}
$$

according to their energy in ascending order. This ensures that the original symbols of interest contained by the desired subvector $\boldsymbol{s}_{\Delta+1}$ are detected first according to the energy of the associated CIR-matrix columns. The corresponding symbols appear in the top section of the search tree.

2) Reorder the remaining columns of the CIR matrix within $\mathbf{H}_{1}$ according to the ascending energy order.

From a physical point of view dispersion-induced lowenergy CIR matrix columns may contribute little to the final

\footnotetext{
${ }^{1}$ If the tree-based feedback method is employed, the symbols are arranged such that they appear directly after the feedback symbols.
} 
cost function value. Nonetheless, the search tree is subject to dispersion-induced branching at its bottom levels due to the low-power, high-delay CIR taps, which have little influence on the final cost function value. These dispersion-induced searchtree sections impose a substantially increased computational complexity, since the cost function contribution of each of these branches has to be evaluated. Due to the fact, that the original high-energy desired symbols appear now in the top section of the search tree, a certain number of low-energy search tree levels at the bottom might be neglected in order to avoid the evaluation of the cost function associated with the fine tree branches induced by the high-delay, low-power CIR taps.

The recursive cost function of Equation (5) can then be rewritten as

$$
J_{i}\left(\check{\mathbf{s}}_{i}\right)=J_{i+1}\left(\check{\mathbf{s}}_{i+1}\right)+\phi\left(\check{\mathbf{s}}_{i}\right), i=N_{\mathrm{s}}-1, \ldots, N_{\text {trunc }}+1,
$$

where $N_{\text {trunc }}$ indicates the number of search tree layers that have been discarded. Note however that this tree-truncation will always result in a controlled performance degradation, because even though we are not interested in the final decision concerning some of the ISI-induced symbols, they still mildly influence the channel's output vector $\mathbf{x}$ and the cost function, as it becomes explicit from Equation (5). However, when the power associated with these ISI-induced symbols is sufficiently low (i.e. the associated column of the channel matrix has a low energy), their influence becomes marginal and hence they may justifyably be neglected.

Let us define $N_{\gamma}$ as the number of columns in the ordered channel matrix, which satisfy

$$
\left\|\left(\mathbf{H}_{1}\right)_{i}\right\|^{2}<\gamma \frac{\sum\left\|\left(\mathbf{H}_{1}\right)\right\|^{2}}{N_{\mathrm{s}}}+2 \sigma_{\mathrm{n}}^{2} .
$$

The number of levels in the search tree, which are neglected is then given by

$$
N_{\text {trunc }}= \begin{cases}N_{\max } & \text { if } N_{\gamma} \geq N_{\max } \\ N_{\gamma} & \text { otherwise }\end{cases}
$$

where $N_{\max }$ has to be carefully chosen for ensuring that the specific levels of the search-tree, which are associated with the desired symbols are not truncated, yielding $N_{\max }=N_{\mathrm{s}}-$ $\log _{2}(\mathcal{M}) M$.

\section{Simulation Results}

Let us now consider a propagation scenario having $M=4$ transmit AEs, $N=2$ receive AEs and a dispersive symbolspaced three-tap CIR characterized by the average path powers of $0 \mathrm{~dB},-3 \mathrm{~dB}$ and $-6 \mathrm{~dB}$, respectively. The parameters of the DF-STE were chosen to be $N_{\mathrm{ff}}=3, \Delta=2$, $N_{\mathrm{fb}}=2$. The modulation scheme considered was 4QAM and independent, uncorrelated Rayleigh fading was assumed. The channel code employed was a half-rate puncutred turbo-code having an interleaver-length of 4000 bits, which was operating on the hard-output of the DF-STE. Even for this modest-size system the full DF-aided ML detector would have to consider $N_{\text {states }}=4^{4 \cdot 3}=16777216$ states for the detection of each bit.

Figure 2(a) shows the average uncoded Bit Error Rate (BER) versus Signal-to-Noise Ratio (SNR) performance of the proposed detector, while Figure 2(b) portrays the associated coded BER. It can be observed from both Figure 2(a) as well as Figure 2(b) that the Tree-Based (TB) and the SpaceTranslation (ST) based DF result in an identical BER performance. If the proposed truncation scheme is applied to the STaided DF-STE, it can be observed that the BER performance is degraded upon increasing the value of $\gamma$. The performance degradation owing to truncation is less pronounced for the coded system of Figure 2(b) than it is for the uncoded system of Figure 2(a).

Let us now finally quantify the computational complexity of the detector in terms of the number of real-valued operations required for the evaluation of Equation (5) and normalized by the $M \log _{2}(\mathcal{M})$ number of bits detected. The average computational cost, which is quantified in Figure 2(c) for different values of the truncation parameter $\gamma$, is significantly decreased in the low-SNR region without a noticeable performance degradation. For higher values of $\gamma$ the computational complexity is also reduced in the high SNR region, albeit it is now associated with a noticeable performance degradation.

When considering the Probability Density Function (PDF) of the computational complexity illustrated in Figure 2(d) for $\mathrm{SNR}=20 \mathrm{~dB}$, it can be observed that tree truncation is capable of substantially reducing the associated complexity. Note that the average complexities at $\mathrm{SNR}=20 \mathrm{~dB}$ illustrated in Figure 2(c) represent the mean values of the associated PDFs illustrated in Figure 2(d). Observe further that as a result of the long tail of the PDFs, the average complexity seen in Figure 2(c) is higher than the abscissa values of the PDF peaks observed in Figure 2(d).

\section{CONCLUSIONS}

The recently proposed OHRSA algorithm [4] has been further developed for employment in a single-carrier STE and has been modified for the sake of accommodating the specific properties of the associated dispersive propagation channel. In order to improve the attainable performance and to reduce the computational complexity of the algorithm we have presented two different ways of incorporating DF. Both proposed DF methods result in a similar BER performance.

A further complexity reduction has been achieved by using a detection tree truncation scheme, which is highly beneficial in propagation environments associated with long-delay, lowpower signal propagation paths. For the example considered in Section VI the full ML detector also benefiting from DF would have to consider $4^{4 \cdot 3}=16777216$ possible channel output states, while the proposed detector only required approximately 2000 operations for the detection of a single bit.

\section{REFERENCES}

[1] A. J. Paulraj, D. A. Gore, R. U. Nabar, and H. Bolcskei, "An overview of MIMO communications - a key to gigabit wireless," Proc. of the IEEE, vol. 92, pp. 198-218, Feb. 2004.

[2] L. Hanzo, T. H. Liew, and B. L. Yeap, Turbo Coding, Turbo Equalisation and Space-Time Coding. Chichester, UK; Piscataway, NJ, USA: John Wiley, IEEE Press, 2002. 766 pages. (For detailed contents, please refer to http://www-mobile.ecs.soton.ac.uk.).

[3] L. Hanzo, M. Münster, B. Choi, and T. Keller, OFDM and MC-CDMA for Broadcasting Multi-User Communications, WLANs and Broadcasting. John Wiley and IEEE Press, July 2003. 


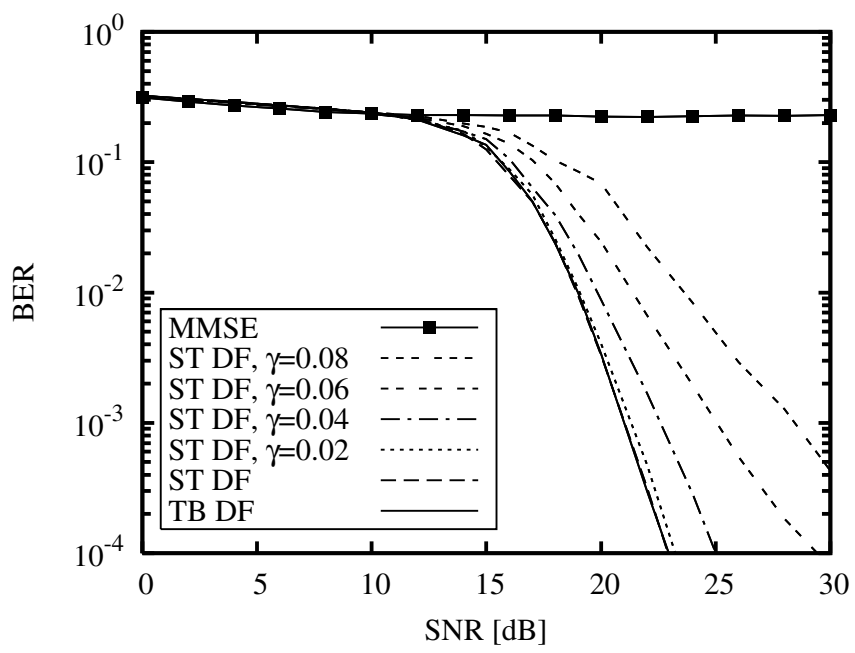

(a) Uncoded BER.

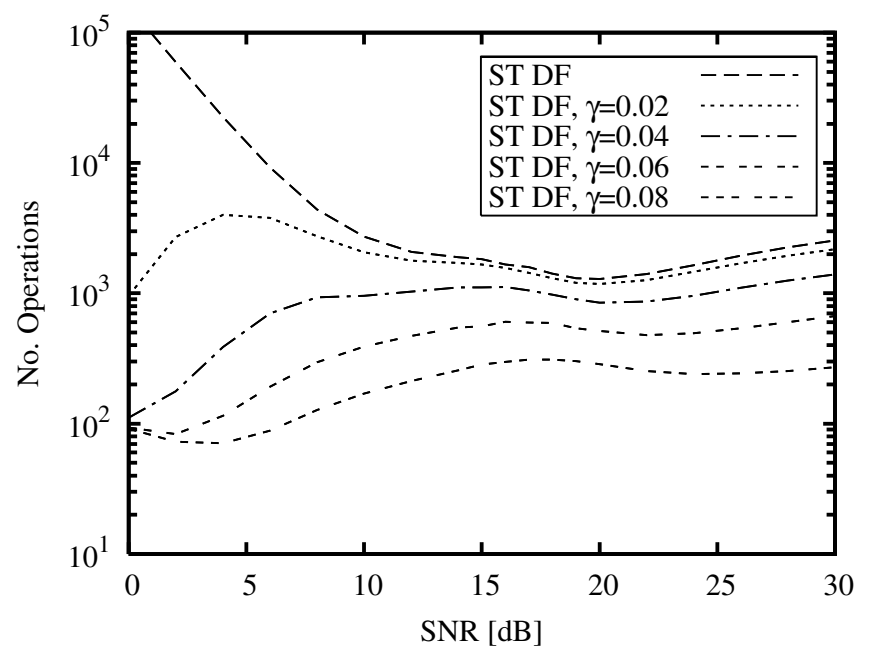

(c) Average computational complexity.

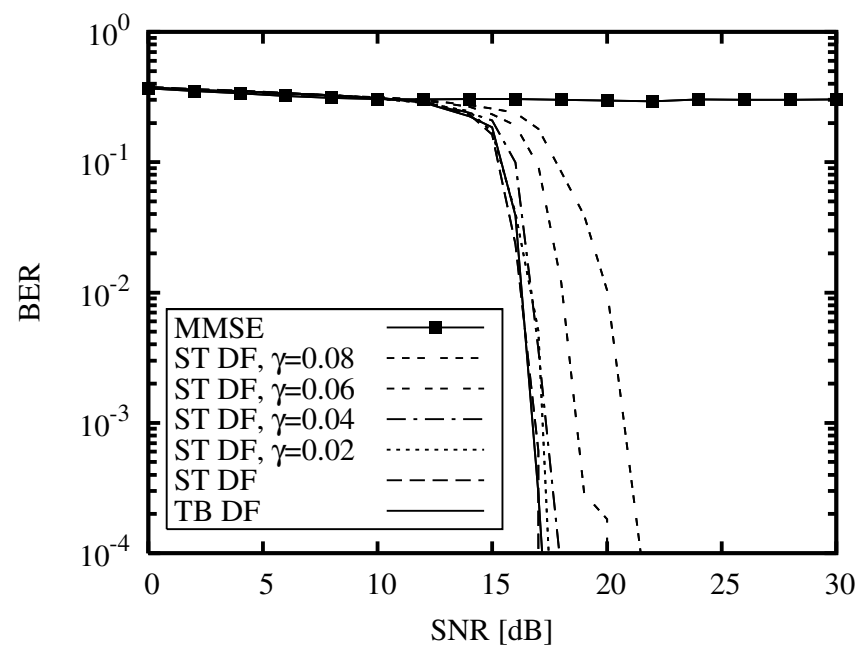

(b) Coded BER.

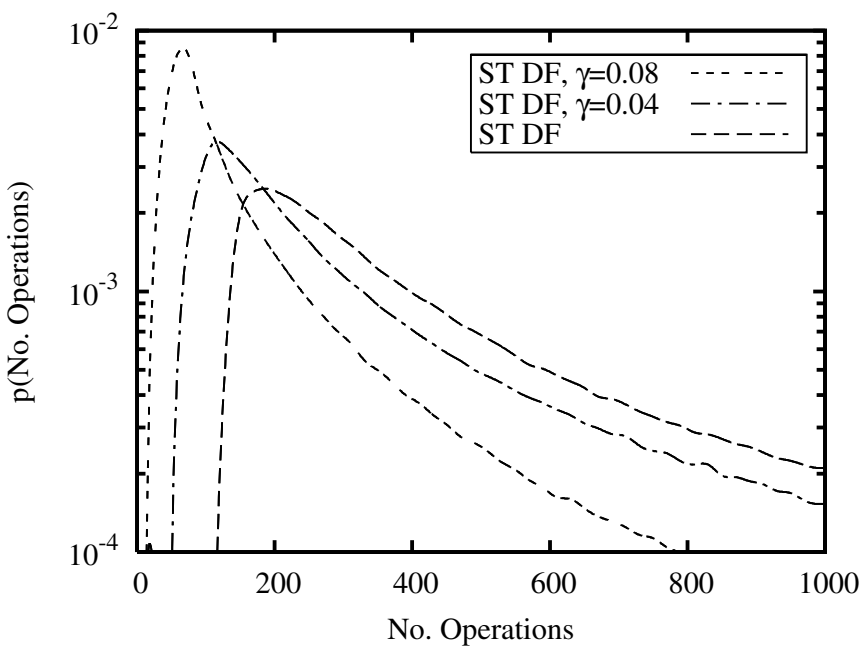

(d) Complexity PDF at $\mathrm{SNR}=20 \mathrm{~dB}$.

Fig. 2. A system having $M=4$ transmit AEs, $N=2$ receive AEs and a DF-STE characterized by $N_{\mathrm{ff}}=3, \Delta=2$ and $N_{\mathrm{fb}}=2$ was considered. The channel was assumed to be an independent, uncorrelated Rayleigh fading channel having a symbol-spaced three-tap CIR characterized by the average path powers of $0 \mathrm{~dB},-3 \mathrm{~dB}$ and $-6 \mathrm{~dB}$, respectively. The channel code employed was a half-rate puncutred turbo-code having an interleaver-length of 4000 bits. The truncation parameter $\gamma$ was chosen to be $0.2,0.4,0.6$ and 0.8 , respectively, as indicated in the labels.

[4] J. Akhtman and L. Hanzo, "Maximum-likelihood enhanced sphere decoding of MIMO-OFDM," in OFDM and MC-CDMA: A Primer by L. Hanzo and T. Keller, pp. 253-301. New York, USA: John Wiley, 2006. (URL: http://www-mobile.ecs.soton.ac.uk/comms/Books.htm).

[5] J. Blogh and L. Hanzo, Third-Generation Systems and Intelligent Networking. John Wiley and IEEE Press, 2002. (For detailed contents, please refer to http://www-mobile.ecs.soton.ac.uk.).

[6] G. Foschini and M. Gans, "On limits of wireless communication in a fading environment when using multiple antennas," Wireless Personal Commun., vol. 6, pp. 311-335, March 1998.

[7] A. Goldsmith, S. J. N., Jindal, and S. Vishwanath, "Capacity limits of MIMO channels," IEEE J. Sel. Areas Commun., vol. 21, no. 5, pp. 684$702,2003$.

[8] C. Tidestav, M. Sternad, and A. Ahlen, "Reuse within a cell-interference rejection or multiuser detection?," IEEE Trans. Commun., vol. 47, pp. 1511-1522, Oct. 1999.

[9] U. Fincke and M. Pohst, "Improved method for calculating vector of short length in a lattice, including a complexity analysis," Math. Comput., vol. 44, pp. 463-471, April 1985.

[10] T. Cui and C. Tellambura, "An efficient generalized sphere decoder for rank-deficient MIMO systems," IEEE Commun. Lett., vol. 9, no. 5, pp. 423-425, 2005.

[11] M. Damen, K. Abed-Meraim, and J.-C. Belfiore, "Generalised sphere decoder for asymmetrical space-time communication architecture," Electron. Lett., vol. 36, no. 2, pp. 166-167, 2000.

[12] H. Artes, "Reducing sphere decoder complexity by elliptical tree pruning," in Proc. 5th IEEE Workshop on Signal Processing Advances in Wireless Communications, pp. 333-337, July 2004.

[13] L. Hanzo, C. H. Wong, and M. S. Yee, Adaptive Wireless Transceivers: Turbo-Coded, Turbo-Equalized and Space-Time Coded TDMA, CDMA, and OFDM Systems. John Wiley and IEEE Press, Feb. 2002.

[14] S. Chen, B. Mulgrew, E.-S. Chng, and G. J. Gibson, "Space translation properties and the minimum-BER linear-combiner DFE," IEE Proc. on Commun., vol. 145, pp. 316-322, Oct. 1998. 